PROCEEDINGS OF THE

AMERICAN MATHEMATICAL SOCIETY

Volume 128, Number 2, Pages 547-556

$\mathrm{S}$ 0002-9939(99)04999-0

Article electronically published on July 6, 1999

\title{
A DISTRIBUTIONAL CONVOLUTION FOR A GENERALIZED FINITE FOURIER TRANSFORMATION
}

\author{
J. J. BETANCOR, M. LINARES, AND J. M. R. MÉNDEZ
}

(Communicated by Palle E. T. Jorgensen)

\begin{abstract}
In this paper we define a generalized finite Fourier transformation in distribution spaces. Also we investigate a distributional convolution for this finite integral transformation.
\end{abstract}

\section{INTRODUCTION}

K. Trimèche [T1] introduced the generalized Fourier transformation

$$
(\mathcal{F} f)(\lambda)=\int_{0}^{\infty} \varphi_{\lambda}(x) f(x) A(x) d x
$$

where (i) $A(t)$ is a real function defined on $[0, \infty)$ such that $A(t)=t^{2 \alpha+1} C(t)$ with $a>-1 / 2$ and $C(t)$ is an even, infinitely differentiable and strictly positive function on $\mathbb{R}$, and (ii) the kernel $\varphi_{\lambda}$ is the solution of the initial value problem

$$
\begin{gathered}
\Delta \varphi=-\lambda^{2} \varphi, \\
\varphi(0)=1, \\
D \varphi(0)=0
\end{gathered}
$$

for every $\lambda \in \mathbb{C}$. In (1) $\Delta$ represents the operator $\frac{1}{A(t)} D(A(t) D)-q(t), D=\frac{d}{d t}$, and the function $q(t)$ is even and infinitely differentiable on $\mathbb{R}$. Besides, he defined and investigated a convolution for the $\mathcal{F}$-transformation.

Later, K. Trimèche [T2] studied generalized Fourier series expansions associated to the operator $\Delta$ when $q(t)=-\gamma^{2}, \gamma \geq 0$. We now recall some of his definitions and results that will be useful in the sequel. Thus, $\mathcal{E}_{*}(\mathbb{R})$ is the space of all even infinitely differentiable functions on $\mathbb{R}$. We assign to $\mathcal{E}_{*}(\mathbb{R})$ the topology generated by the family of seminorms $\left\{p_{n, m}\right\}_{m \in \mathbb{N}}$ where

$$
p_{n, m}(\phi)=\sup _{|x| \leq n}\left|D^{m} \phi(x)\right|, \quad \phi \in \mathcal{E}_{*}(\mathbb{R}),
$$

for every $n, m \in \mathbb{N}$.

The generalized translation operator associated to $\Delta$ is defined for every $\phi \in$ $\mathcal{E}_{*}(\mathbb{R})$ by

$$
\left(\tau_{x} \phi\right)(y)=\chi_{x} \chi_{y}\left[\sigma_{x} \chi^{-1} \phi(y)\right], \quad x, y \in \mathbb{R},
$$

Received by the editors July 12, 1996 and, in revised form, April 7, 1998.

1991 Mathematics Subject Classification. Primary 46F12.

Key words and phrases. Convolution, distribution, Fourier transformation.

(C)1999 American Mathematical Society 
where $\left(\sigma_{x} \phi\right)(y)=\frac{\phi(x+y)+\phi(x-y)}{2}, x, y \in \mathbb{R}$, and $\chi$ represents a generalized RiemannLiouville integral that transmutes the operator $\Delta$ into the operator $D^{2}$ (Definition 5.1 in $[\mathrm{T} 1])$.

Now let $a>0$. Denote by $\mathcal{M}_{a}(\mathbb{R})$ the space of functions $\phi \in \mathcal{E}_{*}(\mathbb{R})$ such that $\left(\tau_{x} \phi\right)(a)=0$, for each $x \in \mathbb{R}$. The space $\mathcal{E}_{*}(\mathbb{R})$ induces on $\mathcal{M}_{a}(\mathbb{R})$ the topology generated by the family $\left\{\alpha_{k}\right\}_{k \in \mathbb{N}}$ of seminorms where for every $k \in \mathbb{N}$

$$
\alpha_{k}(\phi)=\sup _{x \in(0, a)}\left|\Delta^{k} \phi(x)\right|, \quad \phi \in \mathcal{M}_{a}(\mathbb{R}) .
$$

Thus $\mathcal{M}_{a}(\mathbb{R})$ is a Fréchet Montel space. The dual space of $\mathcal{M}_{a}(\mathbb{R})$ will be denoted, as usual, by $\mathcal{M}_{a}(\mathbb{R})^{\prime}$.

Let $\phi, \psi \in \mathcal{M}_{a}(\mathbb{R})$. Then, with the aid of the $\tau$-translation, the convolution $\phi \# \psi$ is defined by

$$
(\phi \# \psi)(x)=\int_{0}^{a}\left(\tau_{x} \phi\right)(y) \psi(y) A(y) d y, \quad x \in \mathbb{R} .
$$

Next, $\left\{ \pm \lambda_{k}\right\}_{k \in \mathbb{N}}$ denote the zeros of the entire function $\lambda \rightarrow \varphi_{\lambda}(a)$ with $\lambda_{0}=0$. From now on $\varphi_{\lambda_{n}}$ will always be represented by $\varphi_{n}$, where $n \in \mathbb{N}$. Note also that $\varphi_{n}$ is in $\mathcal{M}_{a}(\mathbb{R})$, for every $n \in \mathbb{N}$. Then for every $\phi \in \mathcal{M}_{a}(\mathbb{R})$ one has

$$
\phi(x)=\sum_{n=0}^{\infty} \rho_{n} \mathcal{F}(\phi)(n) \varphi_{n}(x)
$$

where the series converges to $\phi$ in $\mathcal{E}_{*}(\mathbb{R})$. Moreover for every $n \in \mathbb{N}$

$$
\rho_{n}=\frac{1}{\int_{0}^{a} \varphi_{n}^{2}(x) A(x) d x}
$$

and

$$
\mathcal{F}(\phi)(n)=\int_{0}^{a} \varphi_{n}(x) \phi(x) A(x) d x .
$$

Let $\phi \in \mathcal{M}_{a}(\mathbb{R})$. The sequence $(\mathcal{F}(\phi)(n))_{n \in \mathbb{N}}$ will be called the generalized finite Fourier transform $\mathcal{F}(\phi)$.

Now some properties are listed:

i) The expression (2) can be seen as an inversion formula for the $\mathcal{F}$-transformation.

ii) Let $\phi, \psi \in \mathcal{M}_{a}(\mathbb{R})$. The convolution takes the form

$$
(\phi \# \psi)(x)=\sum_{n=0}^{\infty} \rho_{n} \mathcal{F}(\phi)(n) \mathcal{F}(\psi)(n) \varphi_{n}(x), \quad x \in \mathbb{R} .
$$

iii) The \#-convolution defines a continuous mapping from $\mathcal{M}_{a}(\mathbb{R}) X \mathcal{M}_{a}(\mathbb{R})$ into $\mathcal{M}_{a}(\mathbb{R})$.

We now consider the space $\mathcal{V}$ of all complex sequences $\left(a_{n}\right)_{n \in \mathbb{N}}$ satisfying

$$
\beta_{k}\left(\left(a_{n}\right)_{n \in \mathbb{N}}\right)=\sum_{n=0}^{\infty} \rho_{n}\left|\lambda_{n}\right|^{2 k}\left|a_{n}\right|<\infty, \quad \text { for every } k \in \mathbb{N} .
$$

The space $\mathcal{V}$ is endowed with the topology associated to the family $\left\{\beta_{k}\right\}_{k \in \mathbb{N}}$ of seminorms.

Proposition 1. The generalized finite Fourier transform is a homeomorphism from $\mathcal{M}_{a}(\mathbb{R})$ onto the space $\mathcal{V}$. 
Proof. It can be proved making use of Theorem 3.I.1, Lemma 3.I.2 and Proposition 3.I.2 $(1,2)$ of $[\mathrm{T} 2]$.

Motivated by the works of A. H. Zemanian [Z1] and R. S. Pathak [P], in this paper we investigate the generalized finite Fourier transformation $\mathcal{F}$ of distributions. In Section 2 we define the generalized finite Fourier transformation on $\mathcal{M}_{a}(\mathbb{R})^{\prime}$. The distributional \#-convolution is studied in Section 3.

Remark 1. Our study includes two important cases. On the one hand, when $A(t)=$ $t^{2 \alpha+1}, \gamma=0$ and $\alpha>-1 / 2, \Delta$ reduces to the Bessel operator. On the other hand, if we choose $A(t)=2^{2(\alpha+\beta+1)}(\sinh t)^{2 \alpha+1}(\cosh t)^{2 \beta+1}, \gamma=\alpha+\beta+1$ and $\alpha, \beta>-1 / 2$, then $\Delta$ turns out to be the Jacobi operator.

Throughout this paper $C$ will always represent a positive constant not necessarily the same in each occurrence.

\section{The Generalized Finite Fourier transformation}

In this section we define the finite generalized Fourier transformation $\mathcal{F}$ on the space $\mathcal{M}_{a}(\mathbb{R})^{\prime}$ and we establish its main properties.

The finite generalized Fourier transform $\mathcal{F}^{\prime}(F)$ of $F \in \mathcal{M}_{a}(\mathbb{R})^{\prime}$ is defined by

$$
\left\langle\mathcal{F}^{\prime}(F),\left(a_{n}\right)_{n \in \mathbb{N}}\right\rangle=\left\langle F(x), \sum_{n=0}^{\infty} \rho_{n} \varphi_{n}(x) a_{n}\right\rangle, \quad\left(a_{n}\right)_{n \in \mathbb{N}} \in \mathcal{V} .
$$

Using well-known results of duality we infer that $\mathcal{F}^{\prime}$ is a homeomorphism from $\mathcal{M}_{a}(\mathbb{R})^{\prime}$ onto $\mathcal{V}^{\prime}$, whenever $\mathcal{M}_{a}(\mathbb{R})^{\prime}$ and $\mathcal{V}^{\prime}$ are endowed either with the strong topology or the weak* topology.

Let $F \in \mathcal{M}_{a}(\mathbb{R})^{\prime}$. There exist $C>0$ and $r \in \mathbb{N}$ such that

$$
|\langle F, \phi\rangle| \leq C \max _{0 \leq k \leq r} \sup _{x \in(0, a)}\left|\Delta^{k} \phi(x)\right|, \quad \phi \in \mathcal{M}_{a}(\mathbb{R}) .
$$

Hence, according to Theorem 1.1 in [T1], we may write

$$
\left|\left\langle F(x), \sum_{n=p}^{\infty} a_{n} \rho_{n} \varphi_{n}(x)\right\rangle\right| \leq C \max _{0 \leq k \leq r} \sum_{n=p}^{\infty}\left|a_{n}\right| \rho_{n}\left|\lambda_{n}\right|^{2 k}
$$

for every $\left(a_{n}\right)_{n \in \mathbb{N}} \in \mathcal{V}$ and $p \in \mathbb{N}$.

Now, by invoking again Proposition 3.I.2 (2) of [T2] we obtain

$$
\left|\left\langle F(x), \sum_{n=p}^{\infty} a_{n} \rho_{n} \varphi_{n}(x)\right\rangle\right| \leq C \sum_{n=p}^{\infty}\left|a_{n}\right|\left|\lambda_{n}\right|^{2 r}\left(\left|\lambda_{n}\right|^{2}+\gamma^{2}\right)^{\alpha+1 / 2},
$$

provided that $p \geq 1$.

Therefore, given an $\varepsilon>0$, there exists a $p_{0} \in \mathbb{N}$ in such a way that

$$
\left|\left\langle F(x), \sum_{n=p}^{\infty} a_{n} \rho_{n} \varphi_{n}(x)\right\rangle\right|<\varepsilon, \quad \text { for every } p \geq p_{0} .
$$

Next, keeping (3) in mind, we can write

$$
\left|\sum_{n=p}^{\infty} a_{n} \rho_{n}\left\langle F, \varphi_{n}\right\rangle\right| \leq C \sum_{n=p}^{\infty}\left|a_{n}\right|\left(\left|\lambda_{n}\right|^{2}+\gamma^{2}\right)^{\alpha+1 / 2}\left|\lambda_{n}\right|^{2 r}<\varepsilon,
$$

provided that $p$ is sufficiently large. 
From (4) and (5) we conclude that

$$
\left\langle F(x), \sum_{n=0}^{\infty} a_{n} \rho_{n} \varphi_{n}(x)\right\rangle=\sum_{n=0}^{\infty} a_{n} \rho_{n}\left\langle F(x), \varphi_{n}(x)\right\rangle, \quad\left(a_{n}\right)_{n \in \mathbb{N}} \in \mathcal{V} .
$$

Thus we have established the following.

Proposition 2. Let $F \in \mathcal{M}_{a}(\mathbb{R})^{\prime}$. Then the sequence $\left(\left\langle F(x), \varphi_{n}(x)\right\rangle\right)_{n \in \mathbb{N}}$ defines a member of $\mathcal{V}^{\prime}$ by

$$
\left\langle\left(\left\langle F(x), \varphi_{n}(x)\right\rangle\right)_{n \in \mathbb{N}},\left(a_{n}\right)_{n \in \mathbb{N}}\right\rangle=\sum_{n=0}^{\infty} \rho_{n}\left\langle F(x), \varphi_{n}(x)\right\rangle a_{n}, \quad\left(a_{n}\right)_{n \in \mathbb{N}} \in \mathcal{V}
$$

and $\mathcal{F}^{\prime}(F)=\left(\left\langle F(x), \varphi_{n}(x)\right\rangle\right)_{n \in \mathbb{N}}$ in the sense of equality in $\mathcal{V}^{\prime}$.

In the sequel we set $\mathcal{F}^{\prime}(F)(n)=\left\langle F(x), \varphi_{n}(x)\right\rangle, n \in \mathbb{N}$ and $F \in \mathcal{M}_{a}(\mathbb{R})^{\prime}$.

Remark 2. If $\phi \in \mathcal{M}_{a}(\mathbb{R})$, we get $\mathcal{F}^{\prime}(\phi)=\left(\left\langle\phi(x), \varphi_{n}(x)\right\rangle\right)_{n \in \mathbb{N}}=(\mathcal{F}(\phi)(n))_{n \in \mathbb{N}}$. Consequently, the classical $\mathcal{F}$-transformation on $\mathcal{M}_{a}(\mathbb{R})$ is a particular case of the $\mathcal{F}^{\prime}$-transformation.

We now show a representation for the elements of $\mathcal{M}_{a}(\mathbb{R})^{\prime}$ that can be seen as an inversion formula for the generalized $\mathcal{F}^{\prime}$-transformation.

Proposition 3. Let $F \in \mathcal{M}_{a}(\mathbb{R})^{\prime}$. Then

$$
F=\lim _{n \rightarrow \infty} \sum_{p=0}^{n} \rho_{p}\left\langle F, \varphi_{p}\right\rangle \varphi_{p}
$$

where the convergence is understood in the strong topology of $\mathcal{M}_{a}(\mathbb{R})^{\prime}$.

Proof. Let $\in \mathcal{M}_{a}(\mathbb{R})^{\prime}$. Note that it is sufficient to prove $\left\{\sum_{p=0}^{n} \rho_{p}\left\langle F, \varphi_{p}\right\rangle \varphi_{p}\right\}_{n \in \mathbb{N}}$ is a weak* convergent sequence to $F$, because $\mathcal{M}_{a}(\mathbb{R})$ is a Montel space.

Let $\phi \in \mathcal{M}_{a}(\mathbb{R})$. According to [T2, Theorem 3.I.1] we get

$$
\phi(x)=\sum_{p=0}^{\infty} \rho_{p} \mathcal{F}(\phi)(p) \varphi_{p}(x), \quad x \in \mathbb{R} .
$$

Moreover the series is convergent in $\mathcal{M}_{a}(\mathbb{R})$. By taking into account that, for every $p \in \mathbb{N}, \varphi_{p}$ defines an element of $\mathcal{M}_{a}(\mathbb{R})^{\prime}$ by

$$
\left\langle\varphi_{p}, \phi\right\rangle=\int_{0}^{a} \varphi_{p}(x) \phi(x) A(x) d x, \quad \phi \in \mathcal{M}_{a}(\mathbb{R}),
$$

it follows that

$$
\begin{aligned}
\langle F, \phi\rangle & =\lim _{n \rightarrow \infty}\left\langle F, \sum_{p=0}^{n} \rho_{p} \mathcal{F}(\phi)(p) \varphi_{p}\right\rangle \\
& =\lim _{n \rightarrow \infty} \sum_{p=0}^{n} \rho_{p} \mathcal{F}(\phi)(p)\left\langle F, \varphi_{p}\right\rangle=\lim _{n \rightarrow \infty}\left\langle\sum_{p=0}^{n} \rho_{p}\left\langle F, \varphi_{p}\right\rangle \varphi_{p}, \phi\right\rangle .
\end{aligned}
$$

Our next objective is to characterize the complex sequences that are $\mathcal{F}^{\prime}$ transforms of an element of $\mathcal{M}_{a}(\mathbb{R})^{\prime}$. First, we need to characterize the elements of $\mathcal{V}^{\prime}$ 
Proposition 4. Let $H$ be a linear functional on $\mathcal{V}$. Then $H \in \mathcal{V}^{\prime}$ if, and only if, there exists a complex sequence $\left(b_{n}\right)_{n \in \mathbb{N}}$ such that

$$
\left|b_{n}\right| \leq C \lambda_{n}^{2 k}, \quad n \in \mathbb{N}, n \geq 1,
$$

for some $C>0$ and some $k \in \mathbb{N}$, for which

$$
\left\langle H,\left(a_{n}\right)_{n \in \mathbb{N}}\right\rangle=\sum_{n=0}^{\infty} b_{n} a_{n} \rho_{n}, \quad\left(a_{n}\right)_{n \in \mathbb{N}} \in \mathcal{V} .
$$

Proof. First, assume that $H$ takes the form (7) where $\left(b_{n}\right)_{n \in \mathbb{N}}$ satisfies (6). Because of Proposition 3.I.2 (2) in [T2] it is not hard to see that $H \in \mathcal{V}^{\prime}$.

Conversely, let $H \in \mathcal{V}^{\prime}$. As is well-known there exists $k \in \mathbb{N}$ such that

$$
\left|\left\langle H,\left(a_{n}\right)_{n \in \mathbb{N}}\right\rangle\right| \leq C \max _{0 \leq p \leq k} \sum_{n=0}^{\infty}\left|\lambda_{n}\right|^{2 p}\left|a_{n}\right|, \quad\left(a_{n}\right)_{n \in \mathbb{N}} \in \mathcal{V} .
$$

Even more, since $\lambda_{n} \neq 0, n \in \mathbb{N}$ and $n \geq 1$, and 0 is not an adherent point of the set $\left\{\lambda_{n}\right\}_{n \in \mathbb{N}}$ we can write

$$
\left|\left\langle H,\left(a_{n}\right)_{n \in \mathbb{N}}\right\rangle\right| \leq C \sum_{n=1}^{\infty}\left|\lambda_{n}\right|^{2 k}\left|a_{n}\right|, \quad\left(a_{n}\right)_{n \in \mathbb{N}} \in \mathcal{V} .
$$

We now introduce the subset of $\mathcal{V}$ defined by

$$
W=\left\{\left(a_{n}\right)_{n \in \mathbb{N}} \in \mathcal{V}: a_{0}=0\right\}
$$

and the mapping

$$
\begin{aligned}
& J: W \longrightarrow J(W) \subset \ell_{1} \\
& \quad\left(a_{n}\right)_{n \in \mathbb{N}} \longrightarrow\left(\lambda_{n}^{2 k} a_{n}\right)_{n \in \mathbb{N}}, n \geq 1 .
\end{aligned}
$$

Here as usual $\ell_{1}$ stands for the space of all those complex sequences $\left(a_{n}\right)_{n \in \mathbb{N}, n \geq 1}$ such that $\sum_{n=1}^{\infty}\left|a_{n}\right|<\infty$. It is obvious that $J$ is one to one. Moreover, by virtue of (8), the linear mapping

$$
\begin{aligned}
& L: J(W) \subset \ell \longrightarrow \mathbb{C} \\
& \quad\left(\lambda_{n}^{2 k} a_{n}\right)_{n=1}^{\infty} \longrightarrow\left\langle H,\left(a_{n}\right)_{n \in \mathbb{N}}\right\rangle
\end{aligned}
$$

is continuous when $J(W)$ is endowed with the topology induced on it by $\ell_{1}$. Therefore, by invoking the Hahn-Banach theorem, $L$ can be extended to $\ell_{1}$ as a member of $\ell_{1}^{\prime}$. Then there exists $\left(\beta_{n}\right)_{n \in \mathbb{N}} \in \ell_{\infty}$ such that

$$
L\left(\lambda_{n}^{2 k} a_{n}\right)_{n=1}^{\infty}=\left\langle H,\left(a_{n}\right)_{n \in \mathbb{N}}\right\rangle=\sum_{n=1}^{\infty} \beta_{n} a_{n} \lambda_{n}^{2 k}, \quad\left(a_{n}\right)_{n \in \mathbb{N}} \in W .
$$

From (9) we can conclude that

$$
\left\langle H,\left(a_{n}\right)_{n \in \mathbb{N}}\right\rangle=a_{n}\left\langle H, e_{0}\right\rangle+\sum_{n=1}^{\infty} \beta_{n} a_{n} \lambda_{n}^{2 k}, \quad\left(a_{n}\right)_{n \in \mathbb{N}} \in \mathcal{V},
$$

where $e_{0}=(1,0, \ldots)$, and the proof is finished by taking $b_{0}=\frac{\left\langle H, e_{0}\right\rangle}{\rho_{0}}$ and $b_{n}=$ $\frac{\beta_{n} \lambda_{n}^{2 k}}{\rho_{n}}, n \in \mathbb{N}, n \geq 1$. 
Corollary 1. Let $\left(b_{n}\right)_{n \in \mathbb{N}}$ be a complex sequence. There exists $F \in \mathcal{M}_{a}(\mathbb{R})^{\prime}$ such that $\mathcal{F}^{\prime}(F)(n)=b_{n}, n \in \mathbb{N}$, if, and only if, there exist $C>0$ and $k \in \mathbb{N}$ such that

$$
\left|b_{n}\right| \leq C\left|\lambda_{n}\right|^{2 k}, \quad n \geq 1, n \in \mathbb{N} .
$$

Proof. Necessity is an immediate consequence of [Z2, Theorem 1.8.1]. In order to establish the sufficiency, we consider a sequence $\left(b_{n}\right)_{n \in \mathbb{N}}$ satisfying $(10)$. Then the series $\sum_{n=0}^{\infty} b_{n} \rho_{n} \varphi_{n}$ converges in the strong topology of $\mathcal{M}_{a}(\mathbb{R})^{\prime}$. By denoting said limit by $F$, from Proposition 3 we can infer that $\mathcal{F}^{\prime}(F)(n)=b_{n}, n \in \mathbb{N}$.

\section{The Generalized CONVOLUtion}

In this section we introduce and investigate a convolution operation in $\mathcal{M}_{a}(\mathbb{R})^{\prime}$.

According to [T2] for every $\phi \in \mathcal{M}_{a}(\mathbb{R})$ we define the translation operator $\tau_{x}$, $x \in \mathbb{R}$, on $\mathcal{M}_{a}(\mathbb{R})$ as

$$
\left(\tau_{x} \phi\right)(y)=\sum_{n=0}^{\infty} \rho_{n} \mathcal{F}(\phi)(n) \varphi_{n}(x) \varphi_{n}(y), \quad x, y \in \mathbb{R} .
$$

It is clear that $\mathcal{F}\left(\tau_{x} \phi\right)(n)=\varphi_{n}(x) \mathcal{F}(\phi)(n), n \in \mathbb{N}$ and $x \in \mathbb{R}$.

Proposition 5. For every $x \in \mathbb{R}, \tau_{x}$ continuously maps $\mathcal{M}_{a}(\mathbb{R})$ into itself.

Proof. Let $x \in \mathbb{R}, \phi \in \mathcal{M}_{a}(\mathbb{R})$ and $k \in \mathbb{N}$. We have

$$
\Delta^{k}\left(\tau_{x} \phi\right)(y)=(-1)^{k} \sum_{n=0}^{\infty} \rho_{n} \mathcal{F}(\phi)(n) \varphi_{n}(x) \lambda_{n}^{2 k} \varphi_{n}(y), \quad x, y \in \mathbb{R} .
$$

Then, from [T2, Proposition 3.I.2 (2)] it is deduced that

$$
\sup _{x \in(0, a)}\left|\Delta^{k}\left(\tau_{x} \phi\right)(y)\right| \leq C \sum_{n=0}^{\infty}\left(\left|\lambda_{n}\right|^{2}+\gamma^{2}\right)^{\alpha+1 / 2}\left|\lambda_{n}\right|^{2 k} \mid \mathcal{F}(\phi(n) \mid .
$$

Hence, since $\mathcal{F}$ is a homeomorphism from $\mathcal{M}_{a}(\mathbb{R})$ onto $\mathcal{V}$, we conclude that $\tau_{x}$ is a continuous mapping from $\mathcal{M}_{a}(\mathbb{R})$ into itself.

Proposition 5 allows us to define the \#-convolution $F \# \phi$ of $F \in \mathcal{M}_{a}(\mathbb{R})^{\prime}$ and $\phi \in \mathcal{M}_{a}(\mathbb{R})$ as follows:

$$
(F \# \phi)(x)=\left\langle F(y),\left(\tau_{x} \phi\right)(y)\right\rangle, \quad x \in \mathbb{R} .
$$

Remark 3. Note that if $f \in \mathcal{M}_{a}(\mathbb{R})$, then $f \in \mathcal{M}_{a}(\mathbb{R})^{\prime}$ according to Proposition 2 . Besides, $f \# \phi$ given by (11) coincides with the classical \#-convolution of $f$ and $\phi$.

Two interesting properties of the \#-convolution are shown in the following assertion.

Proposition 6. (i) For every $F \in \mathcal{M}_{a}(\mathbb{R})^{\prime}$ and $\phi \in \mathcal{M}_{a}(\mathbb{R})$

$$
(F \# \phi)(x)=\sum_{n=0}^{\infty} \rho_{n} \mathcal{F}(\phi)(n) \varphi_{n}(x)\left(\mathcal{F}^{\prime} F\right)(n), \quad x \in \mathbb{R} .
$$

(iii) For every $F \in \mathcal{M}_{a}(\mathbb{R})^{\prime}$, the mapping $\phi \rightarrow F \# \phi$ is continuous from $\mathcal{M}_{a}(\mathbb{R})$ into itself. 
Proof. To see (i) we have to prove that

$$
\begin{aligned}
\left\langle F(y), \sum_{n=0}^{\infty} \rho_{n} \mathcal{F}(\phi)(n) \varphi_{n}(x) \varphi_{n}(y)\right\rangle & \\
= & \sum_{n=0}^{\infty} \rho_{n} \mathcal{F}(\phi)(n)\left\langle F(y), \varphi_{n}(y)\right\rangle \varphi_{n}(x), \quad x \in \mathbb{R},
\end{aligned}
$$

for every $F \in \mathcal{M}_{a}(\mathbb{R})^{\prime}$ and $\phi \in \mathcal{M}_{a}(\mathbb{R})$.

Let $f \in \mathcal{M}_{a}(\mathbb{R})^{\prime}$. As is known there exists $k \in \mathbb{N}$ such that

$$
|\langle F(y), \phi(y)\rangle| \leq C \max _{0 \leq r \leq k} \sup _{y \in(0, a)}\left|\Delta^{r} \phi(y)\right|, \quad \phi \in \mathcal{M}_{a}(\mathbb{R}) .
$$

Hence, for every $p \in \mathbb{N}$ and $x \in \mathbb{R}$,

$$
\begin{aligned}
& \left|\left\langle F(y), \sum_{n=p}^{\infty} \rho_{n} \mathcal{F}(\phi)(n) \varphi_{n}(x) \varphi_{n}(y)\right\rangle\right| \\
& \quad \leq \max _{0 \leq r \leq k} \sup _{y \in(0, a)}\left|\Delta^{r}\left(\sum_{n=p}^{\infty} \rho_{n} \mathcal{F}(\phi)(n) \varphi_{n}(x) \varphi_{n}(y)\right)\right| .
\end{aligned}
$$

By invoking again [T2, Proposition 3.I.2 (2)] one has for every $x \in \mathbb{R}, y \in(0, a)$, $k, p \in \mathbb{N}$,

$$
\sum_{n=p}^{\infty} \rho_{n} \mathcal{F}(\phi)(n)\left|\lambda_{n}\right|^{2 k}\left|\varphi_{n}(x)\right|\left|\varphi_{n}(y)\right| \leq C \sum_{n=p}^{\infty}\left(\left|\lambda_{n}\right|^{2}+\gamma^{2}\right)^{\alpha+1 / 2}\left|\lambda_{n}\right|^{2 k}|\mathcal{F}(\phi)(n)| .
$$

Also by proceeding in a similar way we obtain for every $x \in \mathbb{R}$

$$
\left|\sum_{n=p}^{\infty} \rho_{n} \mathcal{F}(\phi)(n)\left\langle F(y), \varphi_{n}(y)\right\rangle \varphi_{n}(x)\right| \leq C \sum_{n=p}^{\infty}\left(\left|\lambda_{n}\right|^{2}+\gamma^{2}\right)^{\alpha+1 / 2}|\mathcal{F}(\phi)(n)|\left|\lambda_{n}\right|^{2 r} .
$$

Since $\mathcal{F}(\phi) \in \mathcal{V}$, by combining (13), (14) and (15) we can establish (12). (2)].

Part (ii) can be proved taking into account (i) and using [T2, Proposition 3.I.2

We will denote by $\mathcal{L}\left(\mathcal{M}_{a}(\mathbb{R})\right)$ the space of the continuous linear mappings from $\mathcal{M}_{a}(\mathbb{R})$ into itself. We now characterize the elements of $\mathcal{L}\left(\mathcal{M}_{a}(\mathbb{R})\right)$ that commute with $\tau$-translations.

Proposition 7. Let $L \in \mathcal{L}\left(M_{a}(\mathbb{R})\right)$. The following two properties are equivalent.

(i) $L\left(\tau_{x} \phi\right)=\tau_{x} L \phi, \phi \in \mathcal{M}_{a}(\mathbb{R})$ and $x \in \mathbb{R}$.

(ii) There exists $F \in \mathcal{M}_{a}(\mathbb{R})^{\prime}$ such that $L \phi=F \# \phi, \phi \in \mathcal{M}_{a}(\mathbb{R})$.

Proof. Let $L \in \mathcal{L}\left(\mathcal{M}_{a}(\mathbb{R})\right)$ such that $L\left(\tau_{x} \phi\right)=\tau_{x}(L \phi)$ for every $x \in \mathbb{R}$ and $\phi \in$ $\mathcal{M}_{a}(\mathbb{R})$. Define a functional $F$ on $\mathcal{M}_{a}(\mathbb{R})$ by

$$
\langle F, \phi\rangle=\langle\delta, L \phi\rangle, \quad \phi \in \mathcal{M}_{a}(\mathbb{R}),
$$

where $\delta$ represents the Dirac distribution.

It is clear that $F \in \mathcal{M}_{a}(\mathbb{R})^{\prime}$. Moreover, for every $\phi \in \mathcal{M}_{a}(\mathbb{R})$

$$
(F \# \phi)(x)=\left\langle F, \tau_{x} \phi\right\rangle=\left\langle\delta, L\left(\tau_{x} \phi\right)\right\rangle=\left\langle\delta, \tau_{x} L \phi\right\rangle=(L \phi)(x), \quad x \in \mathbb{R},
$$

because $\left\langle\delta, \tau_{x} \phi\right\rangle=\phi(x)$, for every $\phi \in \mathcal{M}_{a}(\mathbb{R})$ and $x \in \mathbb{R}$. 
Conversely, if $L \in \mathcal{L}\left(\mathcal{M}_{a}(\mathbb{R})\right)$ is defined by

$$
L \phi=F \# \phi, \quad \phi \in \mathcal{M}_{a}(\mathbb{R}),
$$

where $F \in \mathcal{M}_{a}(\mathbb{R})^{\prime}$, then by invoking Proposition 6

$$
\begin{aligned}
\tau_{x}(L \phi) & =\tau_{x}(F \# \phi)=\tau_{x}\left(\sum_{n=0}^{\infty} \rho_{n} \mathcal{F}(\phi)(n) \mathcal{F}^{\prime}(F)(n) \varphi_{n}(y)\right) \\
& =\sum_{n=0}^{\infty} \rho_{n} \mathcal{F}(\phi)(n) \mathcal{F}^{\prime}(F)(n) \varphi_{n}(y) \varphi_{n}(x) \\
& =\left\langle F(z), \sum_{n=0}^{\infty} \rho_{n} \mathcal{F}(\phi)(n) \varphi_{n}(z) \varphi_{n}(y) \varphi_{n}(x)\right\rangle \\
& =F \#\left(\tau_{x} \phi\right)=L\left(\tau_{x} \phi\right), \quad x \in \mathbb{R},
\end{aligned}
$$

because the series $\sum_{n=0}^{\infty} \rho_{n} \mathcal{F}(\phi)(n) \varphi_{n}(z) \varphi_{n}(y) \varphi_{n}(x)$ is convergent in $\mathcal{M}_{a}(\mathbb{R})$ for every $x, y \in \mathbb{R}$.

Let $F$ and $G$ be two arbitrary elements of $\mathcal{M}_{a}(\mathbb{R})^{\prime}$. In accordance with the preceding results, it is natural to try to define $F \# G$ by

$$
\langle F \# G, \phi\rangle=\langle F, G \# \phi\rangle, \quad \phi \in \mathcal{M}_{a}(\mathbb{R}) .
$$

From Proposition 6 if $F, G \in \mathcal{M}_{a}(\mathbb{R})^{\prime}$ we have $F \# G \in \mathcal{M}_{a}(\mathbb{R})^{\prime}$. Also Proposition 6 allows us to infer the following.

Proposition 8. For every $F, G \in \mathcal{M}_{a}(\mathbb{R})^{\prime}$

$$
F \# G=\sum_{n=0}^{\infty} \rho_{n}\left(\mathcal{F}^{\prime} F\right)(n)\left(\mathcal{F}^{\prime} G\right)(n) \varphi_{n}
$$

where the convergence of the series is understood in the strong topology in $\mathcal{M}_{a}(\mathbb{R})^{\prime}$.

Proof. By proceeding as in the proof of Proposition 6 , for every $G \in \mathcal{M}_{a}(\mathbb{R})^{\prime}$ it can be established that

$$
(G \# \phi)(x)=\lim _{n \rightarrow \infty} \sum_{p=0}^{n} \rho_{p} \mathcal{F}(\phi)(p)\left(\mathcal{F}^{\prime} G\right)(p) \varphi_{p}(x), \quad \phi \in \mathcal{M}_{a}(\mathbb{R}),
$$

in the sense of convergence in $\mathcal{M}_{a}(\mathbb{R})$.

Hence it follows for each $F, G \in \mathcal{M}_{a}(\mathbb{R})^{\prime}$ that

$$
\begin{aligned}
\langle F \# G, \phi\rangle & =\lim _{n \rightarrow \infty} \sum_{p=0}^{n} \rho_{p}(\mathcal{F} \phi)(p)\left(\mathcal{F}^{\prime} F\right)(p)\left(\mathcal{F}^{\prime} G\right)(p) \\
& =\lim _{n \rightarrow \infty}\left\langle\sum_{p=0}^{n}\left(\mathcal{F}^{\prime} F\right)(p)\left(\mathcal{F}^{\prime} G\right)(p) \rho_{p} \varphi_{p}, \phi\right\rangle, \quad \phi \in \mathcal{M}_{a}(\mathbb{R}) .
\end{aligned}
$$

Thus we have proved that $\left\{\sum_{p=0}^{n} \rho_{p}\left(\mathcal{F}^{\prime} F\right)(p)\left(\mathcal{F}^{\prime} G\right)(p) \varphi_{p}\right\}_{n \in \mathbb{N}}$ is weak* convergent to $F \# G$, as $n \rightarrow \infty$. Since $\mathcal{M}_{a}(\mathbb{R})$ is a Montel space the proof is concluded.

Remark 4. If $f, g \in \mathcal{M}_{a}(\mathbb{R})$, then the generalized convolution $f \# g$ defined by (16) coincides with the classical \#-convolution. 
It is not hard to prove the following properties for the \#-convolution.

Proposition 9. Let $F, G, H \in \mathcal{M}_{a}(\mathbb{R})^{\prime}$. Then:

(i) $F \# G=G \# F$.

(ii) $(F \# G) \# H=F \#(G \# H)$.

(iii) $F \# \delta=F$, where $\delta$ as usual denotes the Dirac distribution.

(iv) $\mathcal{F}^{\prime}(F \# G)(n)=\mathcal{F}^{\prime}(F)(n) \mathcal{F}^{\prime}(G)(n), n \in \mathbb{N}$.

The $\tau$-translation is defined on $\mathcal{M}_{a}(\mathbb{R})^{\prime}$ in a usual way as the transpose of the $\tau$-translation on $\mathcal{M}_{a}(\mathbb{R})$, i.e., for every $F \in \mathcal{M}_{a}(\mathbb{R})^{\prime}$ we define

$$
\left\langle\tau_{x} F, \phi\right\rangle=\left\langle F, \tau_{x} \phi\right\rangle, \quad \phi \in \mathcal{M}_{a}(\mathbb{R}) \text { and } x \in \mathbb{R} .
$$

The space $\mathcal{L}\left(\mathcal{M}_{a}(\mathbb{R})^{\prime}\right)$ denotes the set of continuous linear mappings from $\mathcal{M}_{a}(\mathbb{R})^{\prime}$ into itself when $\mathcal{M}_{a}(\mathbb{R})^{\prime}$ is endowed with the weak* topology.

We now characterize the commuting elements with $\tau_{x}$ of $\mathcal{L}\left(\mathcal{M}_{a}(\mathbb{R})^{\prime}\right)$.

Proposition 10. Let $L \in \mathcal{L}\left(\mathcal{M}_{a}(\mathbb{R})^{\prime}\right)$. The two following properties are equivalent.

(i) $L\left(\tau_{x} G\right)=\tau_{x} L G$, for every $G \in \mathcal{M}_{a}(\mathbb{R})^{\prime}$ and $x \in \mathbb{R}$.

(ii) There exists $F \in \mathcal{M}_{a}(\mathbb{R})^{\prime}$ such that $L G=F \# G$, for every $G \in \mathcal{M}_{a}(\mathbb{R})^{\prime}$.

Proof. To see that (i) implies (ii) we first note that the family $\left\{\tau_{x} \delta\right\}_{x \in \mathbb{R}}$ is a weakly* dense subset of $\mathcal{M}_{a}(\mathbb{R})^{\prime}\left(\left[\mathrm{K}\right.\right.$, Problem W(b)]). Define the mapping $\Omega \in \mathcal{L}\left(\mathcal{M}_{a}(\mathbb{R})^{\prime}\right)$ by

$$
\Omega G=L(G)-G \#(L \delta), \quad G \in \mathcal{M}_{a}(\mathbb{R})^{\prime} .
$$

By invoking Proposition 9 (iii), for every $x \in \mathbb{R}$ we have

$$
\begin{aligned}
\Omega\left(\tau_{x} \delta\right) & =L\left(\tau_{x} \delta\right)-\tau_{x} \delta \#(L \delta) \\
& =\tau_{x} L \delta-\tau_{x}(\delta \# L \delta)=\tau_{x} L \delta-\tau_{x} L \delta=0 .
\end{aligned}
$$

Then $\left\{\tau_{x} \delta\right\}_{x \in \mathbb{R}}$ is contained in the kernel of $\Omega$. Hence we have concluded that $\Omega=0$ and $L G=L \delta \# G, G \in \mathcal{M}_{a}(\mathbb{R})^{\prime}$.

Now let $G \in \mathcal{M}_{a}(\mathbb{R})^{\prime}$ and $x \in \mathbb{R}$. If (ii) holds we can write

$$
\begin{aligned}
\left\langle\tau_{x}(L G), \phi\right\rangle & =\left\langle\tau_{x}(F \# G), \phi\right\rangle=\left\langle F \# G, \tau_{x} \phi\right\rangle \\
& =\left\langle F(y),\left\langle G(z), \tau_{y}\left(\tau_{x} \phi\right)(z)\right\rangle\right\rangle=\left\langle F(y),\left\langle G(z), \tau_{x}\left(\tau_{y} \phi\right)(z)\right\rangle\right\rangle \\
& =\left\langle F(y),\left\langle\left(\tau_{x} G\right)(z),\left(\tau_{y} \phi\right)(z)\right\rangle\right\rangle=\left\langle F \# \tau_{x} G, \phi\right\rangle, \quad \phi \in \mathcal{M}_{a}(\mathbb{R}) .
\end{aligned}
$$

This completes the proof.

\section{REFERENCES}

[J] D. Judge, On Zemanian's distributional eigenfunction transform, J. Math. Anal. Appl. 34 (1971), 187-201. MR 43:909

[K] J. L. Kelley, General topology, D. van Nostrand, Princeton, New Jersey, 1968. MR 16:1136c

[PP] J. N. Pandey and R. S. Pathak, Eigenfunction expansion of generalized functions, Nagoya Math. J. 72 (1978), 1-25. MR 80d:34029

[P] R. S. Pathak, Orthogonal series representations for generalized functions, J. Math. Anal. Appl. 130 (1988), 316-333. MR 89c:46060

[S] H. H. Schaefer, Topological vector spaces, Springer Verlag, New York, 1971. MR 49:7722

[T1] K. Trimèche, Transformation intégrale de Weyl et théorème de Paley-Wiener associés à un opérateur différentiel singulier sur $(0, \infty)$, J. Math. Pures Appl. 60 (1981), 51-98. MR 83i: 47058 
[T2] K. Trimèche, Fonctions moyenne-périodiques associées à un opérateur différentiel singulier sur $(0, \infty)$ et développement en série de Fourier généralisée, J. Math. Pures Appl. 65 (1986), 1-46. MR 88e:47091

[Z1] A. H. Zemanian, Orthonormal series expansions of certain distributions and distributional transform calculus, J. Math. Anal. Appl. 14 (1966), 263-275. MR 35:214

[Z2] A. H. Zemanian, Generalized integral transformations, Dover Publications, New York, 1987. MR 88i: 44007

Departamento de Análisis Matemático, Universidad de la Laguna, 38271 La laguna, Tenerife, Islas Canarias, Spain

E-mail address: jbetanco@ull.es 